\title{
BASE CHANGE ALONG THE FROBENIUS ENDOMORPHISM AND THE GORENSTEIN PROPERTY
}

\author{
PINCHES DIRNFELD
}

\begin{abstract}
Let $R$ be a local ring of positive characteristic and $X$ a complex with nonzero finitely generated homology and finite injective dimension. We prove that if derived base change of $X$ via the Frobenius (or more generally, via a contracting) endomorphism has finite injective dimension then $R$ is Gorenstein.
\end{abstract}

\section{INTRODUCTION}

Kunz [10] proved that a local ring $(R, \mathfrak{m}, k)$ of positive characteristic is regular if and only if some (equivalently, every) power of the Frobenius endomorphism is flat as an $R$-module. Since then analogous characterizations of other properties of the ring, such as complete intersections (by Rodicio 14), Gorenstein (by Goto 16]) and Cohen-Macualay (by Takahashi and Yoshino [15), have been obtained. Many of these results have been established for the larger family of contracting endomorphisms. Following [4, an endomorphism $\varphi: R \rightarrow R$ is said to be contracting if $\varphi^{i}(\mathfrak{m}) \subseteq \mathfrak{m}^{2}$ for some $i>0$. The Frobenius endomorphism is one example but there are many interesting examples even when $R$ is of characteristic 0. Avramov, Iyengar and Miller 4 generalized Kunz's theorem to apply to any contracting endomorphism. For other results concerning contracting endomorphisms see, for example, Avramov, Hochster, Iyengar and Yao [2, Rahmati [13] and Nasseh and Sather-Wagstaff [1].

In this paper we study homological properties of modules and complexes under base change along contracting endomorphisms. Given an endomorpishm $\varphi: R \rightarrow R$, we write $\mathrm{R}^{\varphi}$ for the $R$ bimodule with the right module structure induced by $\varphi$ and the left usual $R$ module structure. Thus given an $R$-complex $X$ the base change along $\varphi$ is $\mathrm{R}^{\varphi} \otimes_{R}^{\mathbf{L}} X$ where $R$ acts on the left through $\mathrm{R}^{\varphi}$. The main result of this work is the following, proved in Section 3 .

Theorem 1.1. Let $\varphi: R \rightarrow R$ be a contracting endomorphism. The following conditions are equivalent.

(i) $R$ is Gorenstein.

(ii) There exists an $R$-complex $X$ with nonzero finitely generated homology and finite injective dimension for which the base change $\mathrm{R}^{\varphi} \otimes_{R}^{\mathrm{L}} X$ has finite injective dimension.

(iii) For every $X$ with nonzero finitely generated homology and finite injective dimension the base change $\mathrm{R}^{\varphi} \otimes_{R}^{\mathbf{L}} X$ has finite injective dimension.

Date: March 24, 2020.

I thank my advisor Srikanth Iyengar for the many helpful discussions and reading many versions of this paper. This work was partly supported by a grant from the National Science Foundation, DMS-1700985. 
An $R$-complex has finite injective dimension if it is quasi-isomorphic to a bounded complex of injective modules.

In the theorem above (i) $\Rightarrow$ (iii) holds because in a Gorenstein local ring complexes of finite injective dimension coincide with complexes of finite projective dimension. For (iii) $\Rightarrow$ (ii) we only need to show that every local ring has a complexes of finite injective dimension with nonzero finitely generated homology. (ii) $\Rightarrow(i)$ is the crucial implication. This is proven in two steps. 1) When $H(X)$ is finitely generated, if $\mathrm{R}^{\varphi^{\mathrm{i}}} \otimes_{R}^{\mathbf{L}} X$ is bounded in homology for $i \gg 0$ then $X$ has finite projective dimension; this follows from well known arguments, see 3.2 for details. 2) When $X$ is a complex with nonzero finite length homology and finite injective dimension, if the base change $\mathrm{R}^{\varphi} \otimes_{R}^{\mathbf{L}} X$ has finite injective dimension then the same holds for $\mathrm{R}^{\varphi^{\mathrm{i}}} \otimes_{R}^{\mathbf{L}} X$ for every $i>0$, see 3.3 . The key tool in the proof of the second step is a theorem of Hopkins 9 and Neeman [12] concerning perfect complexes.

Theorem 1.1 is analogous statement to the following characterization of Gorenstein rings by Falahola and Marley 6

Theorem 1.2. Let $\varphi: R \rightarrow R$ be a contracting endomorphism where $R$ is a CohenMacaulay local ring and $\omega_{R}$ a canonical module. Then $R$ is Gorenstein if and only if $\mathrm{R}^{\varphi} \otimes_{R} \omega_{R}$ has finite injective dimension.

In 6 the authors ask: when $R$ is a local ring with a dualizing complex $D$, if $\mathrm{R}^{\varphi} \otimes R_{R}^{\mathbf{L}} D$ has finite injective dimension is then $R$ necessarily Gorenstein? Theorem 1.1 gives an affirmative answer.

\section{Homological InVARIANTS}

In this Section we recall basic definitions and results for use in Section 3 . Throughout this paper $R$ will be a commutative Noetherian ring. We write $\mathrm{D}(R)$ for the derived category of $R$-complexes, with the convention that complexes are graded below i.e. we write

$$
X=\cdots \rightarrow X_{n} \rightarrow X_{n-1} \rightarrow \cdots
$$

We write $X \simeq Y$ when $X$ is isomorphic to $Y$ in $\mathrm{D}(R)$.

Definition 2.1. Given an $R$-complex $X$ we set

$$
\sup H(X)=\sup \left\{i \mid H_{i}(X) \neq 0\right\} \text { and } \inf H(X)=\inf \left\{i \mid H_{i}(X) \neq 0\right\}
$$

Thus $\sup (0)=-\infty$ and $\inf (0)=\infty$. A complex $X$ is said to be homologically bounded above if $\sup H(X)<\infty$. Similarly, $X$ is homologically bounded below if $\inf H(X)>-\infty$ and $X$ is homologically bounded if it is homologically bounded above and below.

Let $\mathrm{S}$ be a full subcategory of $\mathrm{D}(R)$. We make the following conventions:

(i) $\mathrm{S}_{-}:=\{X \in \mathrm{S} \mid \sup H(X)<\infty\}$

(ii) $\mathrm{S}_{+}:=\{X \in \mathrm{S} \mid \inf H(X)>-\infty\}$

(iii) $\mathrm{S}_{\mathrm{b}}:=\mathrm{S}_{+} \cap \mathrm{S}_{-}$

(iv) The subcategory of of complexes in $\mathrm{S}$ with degree-wise finitely generated (resp. finite length) homology is denoted $\mathrm{S}^{\mathrm{fg}}$ (resp. $\mathrm{S}^{\mathrm{fl}}$ )

In $\mathrm{D}(R)$ we have derived functors $\mathrm{RHom}_{R}(\ldots, \ldots)$ and $\_\otimes_{R}^{\mathbf{L}}$. For a detailed description on how these functors are defined, we refer the reader to [1]. 
When $X$ is in $\mathrm{D}_{+}(R)$, there is a complex $P$ consisting of projective modules with $P_{i}=0$ for $i \ll 0$ such that $P \simeq X$. Such a complex $P$ is called a projective resolution of $X$. In this case we can compute $\operatorname{RHom}\left(X,{ }_{-}\right)$by setting

$$
\operatorname{RHom}\left(X,{ }_{-}\right)=\operatorname{Hom}_{R}(P, \ldots)
$$

Flat and injective resolutions are similarly defined. Since $R$ has enough projectives and injectives, every complex in $\mathrm{D}_{+}(R)$ admits projective (and thereby flat) resolutions and every complex in $\mathrm{D}_{-}(R)$ admits injective resolutions.

Complexes in $\mathrm{D}_{\mathrm{b}}^{\mathrm{fg}}(R)$ with finite projective dimension are the perfect complexes. The subcategory of $\mathrm{D}(R)$ of complexes of finite injective dimension plays a central role in this paper and we denote it $\mathrm{I}(R)$.

Definition 2.2. Let $X \in \mathrm{D}_{+}(R)$ and $Y \in \mathrm{D}_{-}(R)$. We define

$$
\begin{aligned}
& \operatorname{proj-}_{-} \operatorname{dim}_{R}(X):=\inf \left\{\begin{array}{l}
n \mid \begin{array}{c}
\text { there exists a projective resolution } P \text { of } X \\
0 \rightarrow P_{n} \rightarrow \ldots \rightarrow P_{i} \rightarrow 0 \text { with } P_{n} \neq 0
\end{array}
\end{array}\right\}
\end{aligned}
$$

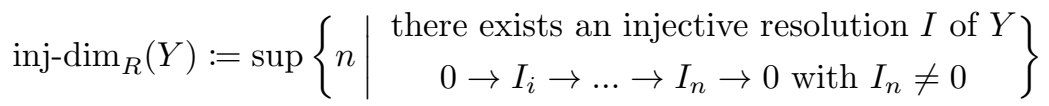

Remark 2.3. Let $(R, \mathfrak{m}, k)$ be a local ring, $X \in \mathrm{D}_{+}(R)$. Following [5, A.5.7, A.7.9] we have what Foxby called "accounting principles".

(i) When $V$ is an R-complex such that $\mathfrak{m} V=0$ then,

$$
\begin{gathered}
\sup H\left(V \otimes_{R}^{\mathbf{L}} X\right)=\sup H(V)+\sup H\left(k \otimes_{R}^{\mathbf{L}} X\right) \\
\inf H\left(V \otimes_{R}^{\mathbf{L}} X\right)=\inf H(V)+\inf H\left(k \otimes_{R}^{\mathbf{L}} X\right)
\end{gathered}
$$

(ii) If $X \in \mathrm{D}_{\mathrm{b}}^{\mathrm{fg}}(R)$ and $Y \in \mathrm{D}_{-}^{\mathrm{fg}}(R)$ then the following hold

$$
\begin{aligned}
\operatorname{proj}_{-} \operatorname{dim}_{R}(X) & =-\inf H\left(\operatorname{RHom}_{R}(X, k)\right)=\sup H\left(k \otimes_{R}^{\mathbf{L}} X\right) \\
\operatorname{inj-}-\operatorname{dim}_{R}(Y) & =-\inf H\left(\operatorname{RHom}_{R}(k, Y)\right)
\end{aligned}
$$

Definition 2.4. A local ring $R$ is Gorenstein if $\operatorname{inj}_{-} \operatorname{dim}_{R}(R)$ is finite.

The following theorem [5, 3.3.4], known as the Foxby equivalence, asserts that in a Gorenstein local ring, the categories of finite projective dimension and finite injective dimension coincide.

Theorem 2.5. Let $(R, \mathfrak{m}, k)$ be a local Gorenstein ring, $X \in \mathrm{D}_{\mathrm{b}}(R)$. Then

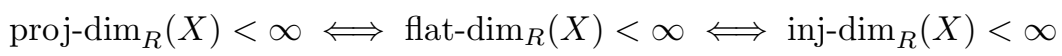

Foxby [7, 2.1] also proved the converse to Theorem 2.5. Although in the original paper it was stated for modules, it is well known to be true for complexes as well. For convenience, we give a self contained proof using the terminology and properties established above.

Theorem 2.6. Let $(R, \mathfrak{m}, k)$ be a local ring. If there exists a complex $X \in \mathrm{D}_{\mathrm{b}}^{\mathrm{fg}}(R)$ with $H(X) \neq 0$ such that both $\operatorname{proj}_{-} \operatorname{dim}_{R}(X)$ and inj-dim $\operatorname{dim}_{R}(X)$ are finite, then $R$ is Gorenstein.

Proof. We have quasi-isomorphisms

$$
\mathrm{RHom}_{R}(k, X) \simeq \operatorname{RHom}_{R}\left(k, R \otimes_{R}^{\mathbf{L}} X\right) \simeq \mathrm{RHom}_{R}(k, R) \otimes_{R}^{\mathbf{L}} X
$$


The first quasi-isomorphism is trivial and the second follows from [7, 1.1.4] since $X$ is perfect. Also, since $X \in \mathrm{D}_{\mathrm{b}}^{\mathrm{fg}}(R)$ we have $\inf H\left(k \otimes_{R}^{\mathbf{L}} X\right)$ is finite. As $H(X) \neq 0$, we get from 2.3 .

$$
\begin{aligned}
\operatorname{inj}_{-} \operatorname{dim}_{R}(X) & =-\inf H\left(\operatorname{RHom}_{R}(k, X)\right) \\
& =-\inf H\left(\operatorname{RHom}_{R}(k, R) \otimes_{R}^{\mathbf{L}} X\right) \\
& =-\inf H\left(\operatorname{RHom}_{R}(k, R)\right)-\inf H\left(k \otimes_{R}^{\mathbf{L}} X\right) \\
& =\operatorname{inj-} \operatorname{dim}_{R} R-\inf H\left(k \otimes_{R}^{\mathbf{L}} X\right)
\end{aligned}
$$

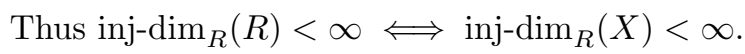

Thick subcategories and generation. Thick subcategories play a critical role in the proofs in Section 3. Here we recall the definition and give some examples, following the formulation given in [3, $\S 1]$

Definition 2.7. A non-empty subcategory $\mathcal{T}$ of $\mathrm{D}(R)$ is thick if it is additive, closed under taking direct summands and for every exact triangle

$$
X \rightarrow Y \rightarrow Z \rightarrow \Sigma X
$$

if any two of $X, Y, Z$ belong to $\mathcal{T}$, so does the third. From the definition it is clear that intersections of thick subcategories is again thick.

Example 2.8. The subcategories $\mathrm{D}^{\mathrm{fg}}(R), \mathrm{D}^{\mathrm{f}}(R), \mathrm{I}(R)$ and the subcategory of perfect complexes are all thick in $\mathrm{D}(R)$, see for example [8, 3.2]. It follows immediately that $\mathrm{I}^{\mathrm{fg}}(R)$ and $\mathrm{I}^{\mathrm{f}}(R)$ are thick as well.

Definition 2.9. The thick subcategory generated by $X \in \mathrm{D}(R)$, $\operatorname{denoted~} \operatorname{Thick}_{R}(X)$, is the smallest thick subcategory that contains $X$. It is the intersection of all thick subcategories of $\mathrm{D}(R)$ containing $X$.

Example 2.10. We always have $\operatorname{Thick}_{R}(R)$ are the perfect complexes. When $(R, \mathfrak{m}, k)$ is local we have $\operatorname{Thick}_{R}(k)=\mathrm{D}_{\mathrm{b}}^{\mathrm{fl}}(R)$.

For any $X \in \mathrm{D}(R)$ one can construct $\operatorname{Thick}_{R}(X)$ as follows: Set $\operatorname{Thick}_{R}^{0}(X)=$ $\{0\}$. The objects of $\operatorname{Thick}_{R}^{1}(X)$ are direct summands of finite direct sums of shifts of $X$. For each $n \geq 2$, the objects of $\operatorname{Thick}_{R}^{n}(X)$ are direct summands of objects $U$ such that $U$ appears in an exact triangle

$$
U^{\prime} \rightarrow U \rightarrow U^{\prime \prime} \rightarrow \Sigma U^{\prime}
$$

where $U^{\prime} \in \operatorname{Thick}_{R}^{n-1}(X)$ and $U^{\prime \prime} \in \operatorname{Thick}_{R}^{1}(X)$. The subcategory $\operatorname{Thick}_{R}^{n}(X)$ is the $n$th thickening of $X$. Every thickening embeds in the next one thus we have a filtration:

$$
\{0\}=\operatorname{Thick}_{R}^{0}(X) \subseteq \operatorname{Thick}_{R}^{1}(X) \subseteq \operatorname{Thick}_{R}^{2}(X) \subseteq \ldots \subseteq \bigcup_{n \geq 0} \operatorname{Thick}_{R}^{n}(X)
$$

It is clear that $\bigcup_{n \geq 0} \operatorname{Thick}_{R}^{n}(X)$ is a thick subcategory. By construction it is the smallest thick subcategory containing $X$ hence

$$
\operatorname{Thick}_{R}(X)=\bigcup_{n \geq 0} \operatorname{Thick}_{R}^{n}(X)
$$

For a broader discussion see, for example, [3, §1]. This discussion motivates the following terminology: An $R$-complex in $\operatorname{Thick}_{R}(X)$ is finitely built from $X$. 
Definition 2.11. The support of an $R$-complex $X$ is

$$
\operatorname{Supp}_{R}(X):=\left\{\mathfrak{p} \in \operatorname{Spec}(R) \mid H(X)_{\mathfrak{p}} \neq 0\right\}
$$

When $X \in \mathrm{D}_{\mathrm{b}}^{\mathrm{fg}}(R)$ the support is

$$
\operatorname{Supp}_{R}(X)=V\left(\operatorname{ann}_{R}(H(X)) .\right.
$$

If $N \in \operatorname{Thick}_{R}(M)$, then from the construction it follows that $\operatorname{Supp}_{R}(N) \subseteq \operatorname{Supp}_{R}(M)$. Indeed, since localization is an exact functor, if $H(M)_{\mathfrak{p}}=0$ for some $\mathfrak{p} \in \operatorname{Spec}(R)$ then inductively $H(N)_{\mathfrak{p}}=0$ for every $N$ in $\operatorname{Thick}_{R}^{i}(M)$ for all $i$.

Hopkins [9, 11] and Neeman [12, 1.2, 2.8] proved the following result which asserts that the converse is true when both $M$ and $N$ are perfect complexes.

Theorem 2.12. Let $R$ be a commutative Noetherian ring. Given perfect $R$-complexes $N$ and $M$, if $\operatorname{Supp}_{R} N \subseteq \operatorname{Supp}_{R} M$ then $N$ is finitely built from $M$.

Loewy Length. Another important element in this work is the Koszul complex. We recall the definition of Koszul complexes and Loewy length.

Definition 2.13. The Koszul complex on $x \in R$ is the $R$-complex

$$
K(x):=0 \rightarrow R \stackrel{x}{\rightarrow} R \rightarrow 0
$$

concentrated in degrees 0 and 1 . Given a sequence $\mathbf{x}=\left(x_{1}, \ldots, x_{n}\right)$ the Koszul complex on $\mathbf{x}$ is

$$
K(\mathbf{x}):=K\left(x_{1}\right) \otimes_{R} K\left(x_{2}\right) \otimes_{R} \cdots \otimes_{R} K\left(x_{n}\right)
$$

with the convention that $K(\varnothing)=R$.

Set $K^{R}$ to be the Koszul complex on a minimal generating set of $\mathfrak{m}$. Since $K^{R}$ is a perfect complex, we have that $K^{R} \in \operatorname{Thick}_{R}(R)$. It follows, that $K^{R} \otimes_{R}^{\mathbf{L}} X$ is in $\operatorname{Thick}_{R}(X)$ for every $X \in \mathrm{D}(R)$.

Definition 2.14. Let $(R, \mathfrak{m}, k)$ be a local ring, $X$ an $R$-complex. The Loewy length of $X$ is defined to be

$$
\ell \ell_{R}(X):=\inf \left\{i \in \mathbb{N} \mid \mathfrak{m}^{i} \cdot X=0\right\}
$$

Following [4, 6.2], the homotopical Loewy length of $X$ is defined to be

$$
\ell \ell_{\mathrm{D}(R)}(X):=\inf \left\{\ell \ell_{R}(V) \mid V \simeq X\right\}
$$

Avramov, Iyengar and Miller [4, 6.2] prove that the homotopical Loewy length satisfies the following finiteness property.

Theorem 2.15. Let $(R, \mathfrak{m}, k)$ be a local ring, and $K^{R}$ be the Koszul complex on a minimal generating set of $\mathfrak{m}$. For any complex $X$ we have

$$
\ell \ell_{\mathrm{D}(R)}\left(K^{R} \otimes_{R}^{\mathrm{L}} X\right) \leq \ell \ell_{\mathrm{D}(R)} K^{R}<\infty
$$

We say that a homomorphism $\varphi:(R, \mathfrak{m}, k) \rightarrow(S, \mathfrak{n}, l)$ is a deep local homomorphism if $\varphi(\mathfrak{m}) \subseteq \mathfrak{n}^{c}$ where $c=\ell \ell_{\mathrm{D}(S)} K^{S}$. The following corollary is often used in the literature to prove various results for deep local homomorphism.

Lemma 2.16. If $\varphi:(R, \mathfrak{m}, k) \rightarrow(S, \mathfrak{n}, l)$ is a deep local homomorphism, then in $\mathrm{D}(R)$ the complex $K^{S}$ is quasi-isomorphic to $H\left(K^{S}\right)$.

Proof. By 2.14 there exist a complex $V$ such that $K^{S} \simeq V$ and $\mathfrak{n}^{c} V=0$. As $\varphi(\mathfrak{m}) \subseteq \mathfrak{n}^{c}$, this yields that $\mathfrak{m} V=0$. Hence the $R$ action on $V$ factors through the map $R \rightarrow R / \mathfrak{m}=k$. Since $k$ is a field, for every $V \in \mathrm{D}(k)$ we have $V \simeq H(V)$. In particular, $K^{S} \simeq H\left(K^{S}\right)$ in $\mathrm{D}(k)$ so the same is true in $\mathrm{D}(R)$. 


\section{Homological Dimension and the Derived Base Change}

Let $\varphi: R \rightarrow S$ be a homomorphism. There is a naturally defined functor $F^{\varphi}$ from the category of $R$-complexes to the category of $S$-complexes by setting

$$
F^{\varphi}\left(\_\right):=S \otimes_{R}
$$

We write

$$
\mathrm{LF}^{\varphi}: \mathrm{D}(R) \rightarrow \mathrm{D}(S) \text { by } \mathrm{LF}^{\varphi}\left(\_\right)=S \otimes_{R}^{\mathrm{L}}-
$$

for the induced functor on $\mathrm{D}(R)$.

Remark 3.1. Let $\varphi:(R, \mathfrak{m}, k) \rightarrow(S, \mathfrak{n}, l)$ be a local homomorphism.

(i) For every perfect complex $X$ the complex $\operatorname{LF}^{\varphi}(X)$ is perfect in $\mathrm{D}(S)$. Indeed, as $X$ is perfect, there exists a finite free resolution $F \simeq X$. Then $\operatorname{LF}^{\varphi}(X) \simeq S \otimes_{R} F$ which is a finite complex of free $S$ modules.

(ii) For every $X \in \mathrm{D}_{+}^{\mathrm{fg}}(R)$ such that $H(X) \neq 0$ we have $H\left(\operatorname{LF}^{\varphi}(X)\right) \neq 0$. Indeed, we may assume $H_{0}(X) \neq 0$ and $H_{i}(X)=0$ for all $i<0$. We have

$$
H_{0}\left(S \otimes_{R}^{\mathbf{L}} X\right) \cong S \otimes_{R} H_{0}(X)
$$

Applying $S \otimes_{R}$ _ to the surjection $H_{0}(X) \rightarrow H_{0}(X) / \mathfrak{m} H_{0}(X) \rightarrow k$ we get $H_{0}\left(S \otimes_{R}^{\mathbf{L}} X\right) \rightarrow S \otimes_{R} k \cong \frac{S}{\mathfrak{m} S} \neq 0$ as $\varphi$ is a local homomorphism.

Proposition 3.2. Let $\varphi:(R, \mathfrak{m}, k) \rightarrow(S, \mathfrak{n}, l)$ be a deep local homomorphism. For any $X \in \mathrm{D}_{+}^{\mathrm{fg}}(R)$ the complex $\mathrm{LF}^{\varphi}(X)$ is homologically bounded above if and only if $X$ has finite projective dimension in $\mathrm{D}(R)$.

Proof. The if part is clear. For the converse, by the disscusion after 2.13 the complex $K^{S} \otimes_{R}^{\mathbf{L}} X \simeq K^{S} \otimes_{S}^{\mathbf{L}}\left(S \otimes_{R}^{\mathbf{L}} X\right)$ is in $\operatorname{Thick}_{S}\left(S \otimes_{R}^{\mathbf{L}} X\right)$. Example 2.8 yields

$$
\sup H\left(S \otimes_{R}^{\mathbf{L}} X\right)<\infty \Longrightarrow \sup H\left(K^{S} \otimes_{R}^{\mathbf{L}} X\right)<\infty .
$$

By Corollary 2.16, the complex $K^{S} \simeq H\left(K^{S}\right)$ in $\mathrm{D}(R)$ and $H\left(K^{S}\right)$ is a $k$-vector space as an $R$-complex, one gets by the Knneth formula

$$
\begin{aligned}
H\left(K^{S} \otimes_{R}^{\mathbf{L}} X\right) & \cong H\left(H\left(K^{S}\right) \otimes_{R}^{\mathbf{L}} X\right) \\
& \cong H\left(H\left(K^{S}\right) \otimes_{k}\left(k \otimes_{R}^{\mathbf{L}} X\right)\right) \\
& \cong H\left(K^{S}\right) \otimes_{k} H\left(k \otimes_{R}^{\mathbf{L}} X\right)
\end{aligned}
$$

Since $H\left(K^{S} \otimes_{R}^{\mathbf{L}} X\right)$ is bounded, so is $H\left(k \otimes_{R}^{\mathbf{L}} X\right)$. Therefore $\operatorname{proj-dim}_{R}(X)<\infty$ by Remark 2.3 (ii).

Corollary 3.3. Let $\varphi:(R, \mathfrak{m}, k) \rightarrow(S, \mathfrak{n}, l)$ be a deep local homomorphism. If there exists an $X \in \mathrm{I}^{\mathrm{fg}}(R)$ with $H(X) \neq 0$ and inj-dim $\operatorname{dim}_{S}\left(\mathrm{LF}^{\varphi}(X)\right)<\infty$ then $R$ is Gorenstein.

Proof. By Remark 3.1(b), the homology $H\left(\operatorname{LF}^{\varphi}(X)\right) \neq 0$. By hypothesis $X$ is in $\mathrm{D}_{\mathrm{b}}^{\mathrm{fg}}(R)$. Hence

$$
\operatorname{inj}_{-} \operatorname{dim}_{S}\left(\operatorname{LF}^{\varphi}(X)\right)<\infty \Longrightarrow \sup H\left(\operatorname{LF}^{\varphi}(X)\right)<\infty
$$

Therefore proj- $\operatorname{dim}_{R}(X)<\infty$ by Proposition 3.2 Theorem 2.6 now shows that $R$ is Gorenstein. 
Remark 3.4. In the context of the Corollary 3.3, if there exists an $X \in \mathrm{D}_{\mathrm{b}}^{\mathrm{fg}}(R)$ with $H(X) \neq 0$ such that inj- $\operatorname{dim}_{R}\left(\operatorname{LF}^{\varphi}(X)\right)<\infty$ then $R$ is regular. Indeed, if $\operatorname{inj}-\operatorname{dim}_{R}\left(\operatorname{LF}^{\varphi}(X)\right)<\infty$ then following the lines of the proof of Proposition 3.2 we see that $\operatorname{inj}-\operatorname{dim}_{R}\left(K^{S} \otimes_{R}^{\mathbf{L}} X\right)<\infty$. It follows that inj- $\operatorname{dim}_{R}\left(k \otimes_{R}^{\mathbf{L}} X\right)$ is finite and therefore $\operatorname{inj}_{-} \operatorname{dim}_{R}(k)<\infty$ which implies that $R$ is regular. This gives another proof of a result of Avramov, Hochster, Iyengar and Yao [2, 5.3].

Our main result concerns the finiteness of injective dimension with respect to the derived base change over contracting endomorphisms.

Definition 3.5. Let $(R, \mathfrak{m}, k)$ be a local ring. An endomorphism $\varphi: R \rightarrow R$ is said to be contracting if $\varphi^{i}(\mathfrak{m}) \subseteq \mathfrak{m}^{2}$ for some $i \geq 1$.

Remark 3.6. If $\varphi$ is a contracting endomorphism then $\varphi^{i}$ will be a deep local homomorphism for each $i \gg 0$.

If $\varphi$ is an endomorphism on $R$, then we define $\mathrm{R}^{\varphi}$ to be $R$ with the right module structure induced by $\varphi$. Proposition 3.2 shows that given a contracting endomorphism $\varphi$ and a complex $X$ then for large enough $i$ the complex $\operatorname{LF}^{\varphi} i(X)$ is bounded if and only if $X$ has finite projective dimension. However, there are examples of complexes of infinite projective dimension for which $\operatorname{LF}^{\varphi}(X)$ is homologically bounded. For example, let

$$
R=\frac{k[x, y]}{\left(x^{3}, y^{3}\right)}
$$

Set $\varphi(x)=y$ and $\varphi(y)=y^{2}$. One can check that $\operatorname{LF}^{\varphi}(x) \simeq(y)$ but $(x)$ has infinite projective dimension. A natural question to ask is when does $\sup H\left(\operatorname{LF}^{\varphi}(X)\right)<\infty$ imply that $\sup H\left(\operatorname{LF}^{\varphi} i(X)\right)<\infty$ for all $i>0$ ? Our goal is to show that if $X$ has finite injective dimension then $\operatorname{LF}^{\varphi}(X)$ is homologically bounded if and only if $\mathrm{LF}^{\varphi}(X)$ is homologically bounded for every $i>0$.

Definition 3.7. Let $(R, \mathfrak{m}, k)$ be a local ring and $E$ the injective hull of the $R$ module $k$. For an $R$-complex $\mathrm{M}$ set

$$
M^{\vee}:=\operatorname{Hom}_{R}(M, E) .
$$

We will need the following lemma; we give a proof for completeness.

Lemma 3.8. Let $(R, \mathfrak{m}, k)$ be a local ring.

(i) The natural map $X \rightarrow X^{\vee \vee}$ is a quasi-isomorphism for all $X \in \mathrm{D}_{\mathrm{b}}^{\mathrm{fl}}(R)$.

(ii) The complex $X^{\vee}$ is perfect with finite length homology for all $X \in \mathrm{I}^{\mathrm{f}}(R)$.

Proof. For (i), we observe that $\left\{X \in \mathrm{D}(R) \mid X \simeq X^{\vee \vee}\right\}$ form a thick subcategory. When $X \in \mathrm{D}_{\mathrm{b}}^{\mathrm{fl}}(R)$ one can show that by induction on the total length of $H(X)$ that $X \in \operatorname{Thick}_{R}(k)$. Clearly $k \simeq k^{\vee \vee}$ so it follows that $X \simeq X^{\vee \vee}$ for all $X \in \mathrm{D}_{\mathrm{b}}^{\mathrm{fl}}(R)$.

For (ii), take an injective resolution $I$ of $X$. Since $\operatorname{Supp}(X)=\{\mathfrak{m}\}$, the injective resolution $I$ is a finite complex where all the modules are direct sums of $E$. Hence by Matlis duality, $X^{\vee}$ is quasi-isomorphic to a bounded complex of free $\widehat{R}$ modules. As $\widehat{R}$ is flat,

$$
\sup \left(k \otimes_{R}^{\mathbf{L}} X^{\vee}\right)=\sup \left(k \otimes_{R}^{\mathbf{L}} X^{\vee} \otimes_{R}^{\mathbf{L}} \widehat{R}\right)
$$

Since $X^{\vee}$ is perfect in $\mathrm{D}(\widehat{R})$ it is also perfect in $\mathrm{D}(R)$.

Proposition 3.9. For all $X \in \mathrm{I}^{\mathrm{f}}(R)$ with $H(X) \neq 0$, one has $\operatorname{Thick}_{R}(X)=\mathrm{I}^{\mathrm{f}}(R)$. 
Proof. By Example 2.8, Thick ${ }_{R}(X) \subseteq \mathrm{I}^{\mathrm{fl}}(R)$, so it suffices to show that for all $Y \in \mathrm{I}^{\mathrm{fl}}(R)$ we have $Y$ is in $\operatorname{Thick}_{R}(X)$. Let $Y \in \mathrm{I}^{\mathrm{f}}(R)$ with $H(Y) \neq 0$. By Lemma 3.8(ii) $X^{\vee}$ and $Y^{\vee}$ are complexes with finite length homology and finite projective dimension over $R$. In particular, $X^{\vee}$ and $Y^{\vee}$ are both perfect, and

$$
\operatorname{Supp}\left(X^{\vee}\right)=\{\mathfrak{m}\}=\operatorname{Supp}\left(Y^{\vee}\right)
$$

Theorem 2.12 yields that

$$
\operatorname{Thick}_{R}\left(X^{\vee}\right)=\operatorname{Thick}_{R}\left(Y^{\vee}\right)
$$

Applying Matlis duality again, and noting that $X^{\vee \vee} \simeq X$ by Lemma 3.8(i), we see that $\operatorname{Thick}_{R}(X)=\operatorname{Thick}_{R}(Y)$. In particular, $Y$ is in $\operatorname{Thick}_{R}(X)$.

Lemma 3.10. Let $\varphi:(R, \mathfrak{m}, k) \rightarrow(R, \mathfrak{m}, k)$ be contracting endomorphism. If for some $X \in \mathrm{I}^{\mathrm{f}}(R)$ with $H(X) \neq 0$ the injective dimension of $\mathrm{LF}^{\varphi}(X)$ is finite then the injective dimension of $\mathrm{LF}^{\varphi^{i}}(Y)$ is finite for all $i \geq 1$ and all $Y \in \mathrm{I}^{\mathrm{fl}}(R)$.

Proof. By Remark 3.1 $H\left(\operatorname{LF}^{\varphi}(X)\right) \neq 0$ when $H(X) \neq 0$. Proposition 3.9] shows that $\operatorname{Thick}_{\mathrm{D}(R)}(X)=\mathrm{I}^{\mathrm{fl}}(R)$. Since $\mathrm{LF}^{\varphi}\left(\_\right)$is an exact functor it follows that $\mathrm{LF}^{\varphi}(Y)$ is in Thick $R\left(\mathrm{LF}^{\varphi}(X)\right)$ for every $Y \in \mathrm{I}^{\mathrm{f}}(R)$. By hypothesis $\mathrm{LF}^{\varphi}(X) \in \mathrm{I}^{\mathrm{f}}(R)$, hence the functor $\mathrm{LF}^{\varphi}\left(\_\right)$takes $\mathrm{I}^{\mathrm{f}}(R)$ to $\mathrm{I}^{\mathrm{f}}(R)$, but this implies that $\mathrm{LF}^{\varphi}(Y) \cong \mathrm{LF}^{\varphi}\left(\mathrm{LF}^{\varphi}(Y)\right)$ has finite injective dimension for every $Y \in \mathrm{I}^{\mathrm{f}}(R)$. By induction on $i$, we have $\mathrm{LF}^{\varphi^{i}}(Y)$ is finite for all $i \geq 1$ and all $Y \in \mathrm{I}^{\mathrm{fl}}(R)$.

The following theorem is a restatement of Theorem 1.1.

Theorem. Let $\varphi:(R, \mathfrak{m}, k) \rightarrow(R, \mathfrak{m}, k)$ be a contracting endomorphism. The following are equivalent.

(i) $R$ is Gorenstein.

(ii) There exists an $X \in \mathrm{I}^{\mathrm{fg}}(R)$ with $H(X) \neq 0$ and $\mathrm{LF}^{\varphi}(X) \in \mathrm{I}^{\mathrm{fg}}(R)$.

(iii) For every $X \in \mathrm{I}^{\mathrm{fg}}(R)$ we have $\mathrm{LF}^{\varphi}(X) \in \mathrm{I}^{\mathrm{fg}}(R)$.

Proof. (i) $\Longrightarrow$ (iii). Theorem 2.5 shows that $\mathrm{I}^{\mathrm{fg}}(R)$ are the perfect complexes. So for every $X \in \mathrm{I}^{\mathrm{fg}}(R)$ the base change $\mathrm{LF}^{\varphi}(X)$ is also perfect and hence in $\mathrm{I}^{\mathrm{fg}}(R)$.

(iii) $\Longrightarrow$ (ii). We need to show that for every local ring there exists a complex $X \in \mathrm{I}^{\mathrm{fg}}(R)$ with $H(X) \neq 0$. Let $E$ be the injective hull of the residue field, $K^{R}$ the Koszul complex of $R$. The complex $K^{R} \otimes_{R} E$ is Artinian and $\mathfrak{m} H\left(K^{R} \otimes_{R} E\right)=0$, hence it has finite length homology.

(ii) $\Longrightarrow$ (i), Let $K^{R}$ be the Koszul complex of $R$. Let $X \in \mathrm{f}^{\mathrm{fg}}(R)$ with

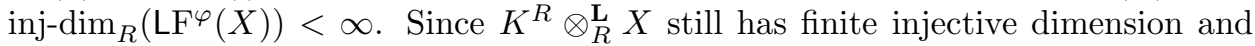
non-zero finite length homology, Lemma 3.10 shows that

$$
\operatorname{inj}_{-\operatorname{dim}_{R}}\left(\operatorname{LF}^{\varphi} i\left(K^{R} \otimes_{R}^{\mathrm{L}} X\right)\right)<\infty \text { for all } i \geq 1
$$

Setting $c=\ell \ell_{\mathrm{D}(R)} K^{R}$ we can take $i$ large enough so that $\varphi^{i}(\mathfrak{m}) \subseteq \mathfrak{m}^{c}$. Corollary 3.3 yields that $R$ is Gorenstein.

Theorem 1.1 is the derived analogue of the following result by Falahola and Marley. [6, Theorem 3.1]

Theorem 3.11. Let $(R, \mathfrak{m}, k)$ be a Cohen-Macaulay local ring, $\varphi$ be a contracting endomorphism. Suppose that $\omega_{R}$ is a canonical module for $R$, then $\operatorname{inj-} \operatorname{dim}_{R_{\varphi}} F^{\varphi}\left(\omega_{R}\right)$ in finite if and only if $R$ is Gorenstein. 
Remark 3.12. Falahola and Marley [6, Example 3.8] show that Theorem 3.11 fails if we replace $\omega_{R}$ with a general dualizing complex $C$. They ask [6, Question 3.9], if $R$ has a dualizing complex $C$ is it true that inj- $\operatorname{dim}_{R}\left({ }^{\varphi} R \otimes_{R}^{\mathbf{L}} C\right)<\infty$ if and only if $R$ is Gorenstein? Since a dualizing complex is in $\mathrm{I}^{\mathrm{fg}}(R)$, Theorem 1.1 shows in particular that if $C$ is a dualizing complex in $\mathrm{D}(R)$ then inj-dim ${ }_{R}\left(\operatorname{LF}^{\varphi}(C)\right)$ being finite implies that $R$ is Gorenstein, giving an affirmative answer.

\section{REFERENCES}

[1] Luchezar L. Avramov and Hans-Bjørn Foxby, Homological dimensions of unbounded complexes, J. Pure Appl. Algebra 71 (1991), no. 2-3, 129-155, DOI 10.1016/0022-4049(91)90144Q. MR1117631

[2] Luchezar L. Avramov, Melvin Hochster, Srikanth B. Iyengar, and Yongwei Yao, Homological invariants of modules over contracting endomorphisms, Math. Ann. 353 (2012), no. 2, 275291, DOI 10.1007/s00208-011-0682-z. MR2915536

[3] Luchezar L. Avramov and Srikanth B. Iyengar, Cohomology over complete intersections via exterior algebras, Triangulated categories, London Math. Soc. Lecture Note Ser., vol. 375, Cambridge Univ. Press, Cambridge, 2010, pp. 52-75. MR2681707

[4] Luchezar L. Avramov, Srikanth Iyengar, and Claudia Miller, Homology over local homomorphisms, Amer. J. Math. 128 (2006), no. 1, 23-90. MR2197067

[5] Lars Winther Christensen, Gorenstein dimensions, Lecture Notes in Mathematics, vol. 1747, Springer-Verlag, Berlin, 2000. MR1799866

[6] Brittney Falahola and Thomas Marley, Characterizing Gorenstein rings using contracting endomorphisms, Journal of Algebra, posted on 2018, DOI 10.1016/j.jalgebra.2018.09.035.

[7] Hans-Bjørn Foxby, Isomorphisms between complexes with applications to the homological theory of modules, Math. Scand. 40 (1977), no. 1, 5-19, DOI 10.7146/math.scand.a-11671. MR0447269

[8] W. Dwyer, J. P. C. Greenlees, and S. Iyengar, Finiteness in derived categories of local rings, Comment. Math. Helv. 81 (2006), no. 2, 383-432, DOI 10.4171/CMH/56. MR2225632

[9] Michael J. Hopkins, Global methods in homotopy theory, Homotopy theory (Durham, 1985), London Math. Soc. Lecture Note Ser., vol. 117, Cambridge Univ. Press, Cambridge, 1987, pp. 73-96. MR932260

[10] Ernst Kunz, Characterizations of regular local rings of characteristic p, Amer. J. Math. 91 (1969), 772-784, DOI 10.2307/2373351. MR252389

[11] Saeed Nasseh and Sean Sather-Wagstaff, Contracting endomorphisms and dualizing complexes, Czechoslovak Math. J. 65(140) (2015), no. 3, 837-865, DOI 10.1007/s10587-0150212-3. MR3407609

[12] Amnon Neeman, The chromatic tower for $D(R)$, Topology 31 (1992), no. 3, 519-532, DOI 10.1016/0040-9383(92)90047-L. With an appendix by Marcel Bökstedt. MR1174255

[13] Hamidreza Rahmati, Contracting endomorphisms and Gorenstein modules, Arch. Math. (Basel) 92 (2009), no. 1, 26-34, DOI 10.1007/s00013-008-2681-1. MR2471985

[14] Antonio G. Rodicio, On a result of Avramov, Manuscripta Math. 62 (1988), no. 2, 181-185, DOI 10.1007/BF01278977. MR963004

[15] Ryo Takahashi and Yuji Yoshino, Characterizing Cohen-Macaulay local rings by Frobenius maps, Proc. Amer. Math. Soc. 132 (2004), no. 11, 3177-3187, DOI 10.1090/S0002-9939-0407525-2. MR2073291

[16] Shiro Goto, A problem on Noetherian local rings of characteristic p, Proc. Amer. Math. Soc. 64 (1977), no. 2, 199-205, DOI 10.2307/2041427. MR447212

Pinches Dirnfeld, Department Of Mathmatics, University of Utah, 155 South 1400

East, JWB 233, Salt Lake City, UT 84112

E-mail address: dirnfeld@math.utah.edu 